\title{
The Path from AMANDA to IceCube
}

\author{
Albrecht Karle \\ Department of Physics and Wisconsin IceCube Particle Astrophysics Center, University of \\ Wisconsin-Madison, Madison, WI 53706, USA
}

\begin{abstract}
In May 2011, IceCube, a neutrino telescope with one cubic kilometer instrumented volume started full operation with 5,160 sensors. The plan to build an experiment of this scale was based in part on the successful realization of a prototype experiment, the Antarctic Muon and Neutrino Detector Array. Here, we will review some of the major challenges and milestones.
\end{abstract}

Keywords. Neutrino

\section{Introduction}

In May 2011, IceCube, a neutrino telescope with one cubic kilometer instrumented volume started full operation with 5,160 sensors. The plan to build an experiment of this scale was based on a decade of research and the demonstration that ice was a suitable medium. The vision for neutrino astronomy was laid out in the early 1960s. After some pioneering efforts to build neutrino detectors in water, similar efforts were staged at the South Pole to build and deploy a Cherenkov neutrino detector. First, in the 1990s, the Antarctic Muon and Neutrino Detector Array (AMANDA) was built. Then, based on AMANDA as a proof of concept, the full kilometer-scale IceCube neutrino telescope was constructed and completed by 2010 (see Fig. 1). Today, the South Pole has become the premier site for neutrino astronomy. In a historical perspective, the idea to use neutrinos for astronomy was born not long after the neutrino was discovered by Reines and collaborators in 1956. For that, a very large target volume was needed, on the order of one cubic kilometer. The other requirement for Cherenkov detectors is a good transparency of the medium. A detailed review of the current state of neutrino astronomy was provided by Spiering \& Katz (2012). Here we will focus on neutrino astronomy at the South Pole.

\section{Overview}

In the 1990-91 austral season, the first exploratory effort was made at the South Pole to deploy photomultipliers in ice at a shallow depth. This would be the first of 13 polar seasons that involved hot water drilling with the goal of deploying photomultipliers in ice and advancing AMANDA and later IceCube. It was preceded by an important exploration of the idea to deploy PMTs in natural ice in Greenland in 1990. The result, the 'Observation of muons using the polar ice cap as a Cerenkov detector' was published by Lowder et al. (Nature, 1991) and marks an important milestone. The authors conclude "Our results suggest that a full-scale Antarctic ice detector is technically quite feasible," and preparations began for an exploration at the South Pole.

A first test array with 80 optical modules on four strings (not shown) was deployed in 1993-94, at depths between 800 and 1,000 $\mathrm{m}$. While the absorption length of blue light was determined to be exceptionally large, on the order of $300 \mathrm{~m}$, it was found that the effective scattering length $L_{\text {eff }}$ was extremely small, between $40 \mathrm{~cm}$ at $830 \mathrm{~m}$ depth and $80 \mathrm{~cm}$ 


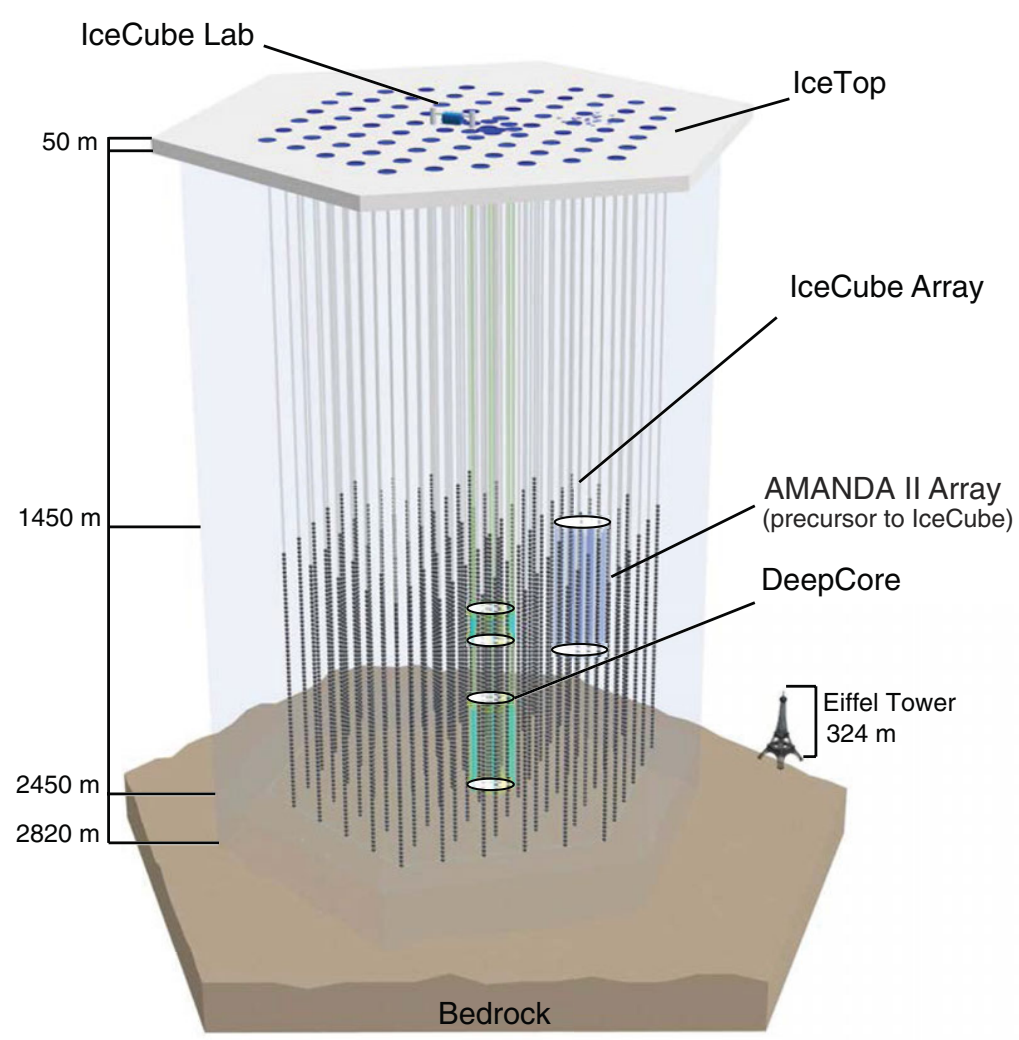

Figure 1. Schematic view of IceCube.

at $970 \mathrm{~m}$ (Askebjer et al. 1995). At these depths, scattering of light is caused primarily by air bubbles trapped in the ice. The density and size of the air bubbles decreases with increasing pressure and age of the ice at greater depths. This trend, together with evidence from ice cores at other locations, suggested that below about 1,350 $\mathrm{m}$ air bubbles disappear and that the air is absorbed in ice clathrate crystals. This was confirmed with a second 4-string array which was deployed in 1995-96. The remaining scattering, averaged over 1,500 to 2,000 $\mathrm{m}$ depth, corresponds to $L_{e f f} \approx 20 \mathrm{~m}$ and is caused primarily by dust. The absorption length was still very good, with more than $100 \mathrm{~m}$. The ice was suitable for the reconstruction of muon tracks and thus for neutrino astronomy.

Over the course of three more construction seasons, the AMANDA-II array was gradually completed by the 1999-2000 season. Already with data from the 10-string configuration completed in 1997 (AMANDA-B10), the collaboration was able to demonstrate detection of atmospheric neutrinos consistent with expectations, an important milestone. There were 188 upward-going muons from neutrinos found in a livetime of 138 days, a little more than one event per day. Figure 2 shows a graphical depiction of the 10-string configuration together with an observed muon neutrino. The result marked another big milestone that was documented in a publication in Nature (Andres et al. 2001). In the meantime, by 2000, the full AMANDA-II array was completed and in operation. The collaboration had grown to more than 20 institutions worldwide with an author list exceeding 100.

The completed AMANDA-II array was a fully functioning neutrino telescope consisting of 19 strings and 677 photomultipliers of $20 \mathrm{~cm}$ diameter. In seven years of operation 


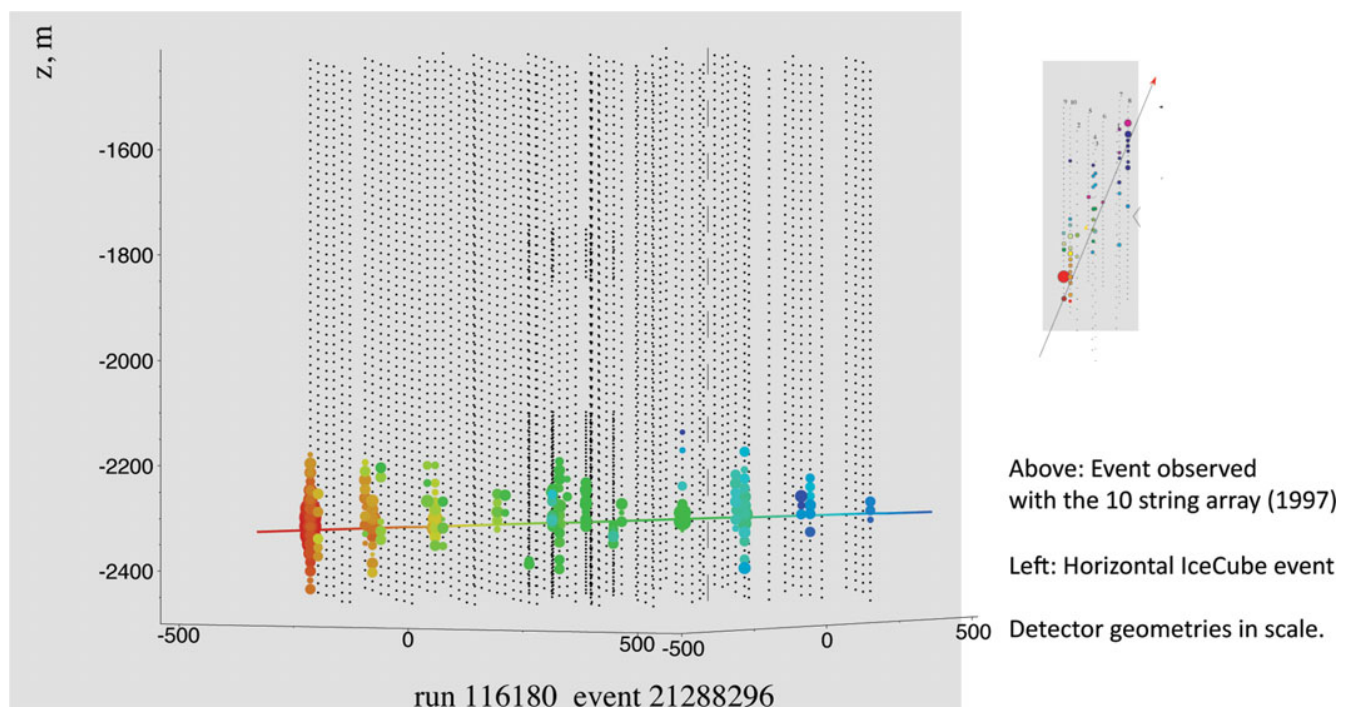

Figure 2. A horizontal muon event of energy $10 \mathrm{TeV}$ recorded with IceCube. On the right: one of the first upward-going muon events observed with the AMANDA 10-string array set to scale.

it collected 6,595 neutrino events in the search for point sources of neutrinos, about 5 events/day. The drilling and installation process was sufficiently developed that plans could be made for IceCube. The proposal for IceCube was submitted in 1999, before AMANDA-II was completed. The experience gained with respect to improved drilling, detector module design and data analysis with the full AMANDA-II would still be critical in preparation for IceCube. It is important to mention string 18, which was instrumented with a prototype of the Digital Optical Module (DOM). The prototype DOM digitized the waveforms and provided an in-situ demonstration of the concept which was then chosen for IceCube's design. The sensors were hybrid modules with simultaneous analog transmission via multimode optical fibers, the latter being the baseline for AMANDA-II.

The Digital Optical Module 3 is built around a $25 \mathrm{~cm}$ diameter hemispherical photomultiplier. Electronics in the sensor are designed to digitize and time stamp the signals. Cherenkov light signals are recorded over a dynamic range from 1 to more than 10,000 photoelectrons. All sensors are synchronized with the master clock to a precision of 1 nsec, a resolution comparable to the spacial extension of the sensors. Each module is equipped with 12 on-board blue LEDs which can be used for verification of spatial detector geometry and timing system as well as for precise measurements of the optical properties of the ice. A glass pressure housing protects the sensor from pressures of up to 500 bar recorded in the deep ice and during the freeze-in process. There are 60 sensors connected to each of the 86 cables that provide power and communication. Maintenance of the full detector was an important consideration for the technology choice. IceCube DOMs and data acquisition system are designed to allow for automatic self-calibration of important parameters at the start of each run. The power consumption of each sensor with its more than 1,000 electronic parts and built in high voltage supply is about $5 \mathrm{~W}$. The system power for IceCube is around $55 \mathrm{~kW}$.

IceCube construction began in earnest in 2004-2005. The commissioning of the more powerful 5-MW IceCube hot water drill proved to be a challenge. The system gathered data from about 300 electronic sensors, and all pumps, heaters and valves were computer controlled and had safety systems. Safety was a big challenge when melting and 


\begin{tabular}{|c|c|c|c|c|c|c|}
\hline Season & Campaign & $\begin{array}{l}\text { Sensors } \\
\text { cum. }\end{array}$ & Strings & $\begin{array}{c}\text { Depth } \\
(\mathrm{m})\end{array}$ & $\begin{array}{l}\text { Neutrinos } \\
\text { per day }\end{array}$ & $\begin{array}{c}\text { Resol. } \\
@ 100 \mathrm{TeV}\end{array}$ \\
\hline 1991-1992 & Exploratory & few & & Shallow & - & \\
\hline \multicolumn{7}{|l|}{$1992-1993$} \\
\hline 1993-1994 & AMANDA-A & 80 & 4 & $800-1000$ & - & \\
\hline \multicolumn{7}{|l|}{ 1994-1995 } \\
\hline 1995-1996 & AMANDA-B4 & 86 & 4 & $1500-1950$ & $\sim 0.01$ & \\
\hline 1996-1997 & AMANDA-B10 & 206 & $6 / 10$ & $1500-1950$ & $\sim 1$ & $4^{\circ}$ \\
\hline \multicolumn{7}{|l|}{ 1997-1998 } \\
\hline 1998-1999 & AMANDA-II & 306 & $3 / 13$ & $1500-1950$ & & \\
\hline 1999-2000 & AMANDA-II & 677 & $6 / 19$ & $1500-1950$ & $\sim 5$ & $2^{\circ}$ \\
\hline \multicolumn{7}{|l|}{$2001-2002$} \\
\hline \multicolumn{7}{|l|}{$2002-2003$} \\
\hline $2003-2004$ & IceCube prep. & & & & & \\
\hline $2004-2005$ & IceCube 1 & 60 & $1 / 1$ & $1450-2450$ & & \\
\hline 2005-2006 & IceCube 9 & 540 & $8 / 9$ & $1450-2450$ & & \\
\hline $2006-2007$ & IceCube 22 & 1320 & $13 / 22$ & $1450-2450$ & 18 & $1.5^{\circ}$ \\
\hline $2007-2008$ & IceCube 40 & 2400 & $18 / 40$ & $1450-2450$ & 40 & $0.8^{\circ}$ \\
\hline 2008-2009 & IceCube 59 & 3540 & $19 / 59$ & $1450-2450$ & 120 & $0.6^{\circ}$ \\
\hline 2009-2010 & IceCube 79 & 4740 & $20 / 79$ & $1450-2450$ & 180 & $0.4^{\circ}$ \\
\hline $2010-2011$ & IceCube 86 & 5160 & $7 / 86$ & $1450-2450$ & $>200$ & $0.4^{\circ}$ \\
\hline
\end{tabular}

Table 1. The table summarizes the deployment of optical sensors at the South Pole. The cumulative number of sensors deployed per year is shown (324 IceTop sensors deployed with IceCube are not included). The angular resolution is shown for the reference analysis for point source searches.

circulating more than $800 \mathrm{l} / \mathrm{min}$ of hot water at $90^{\circ} \mathrm{C}$ and at a pressure of $70 \mathrm{bar}$. The drill head was $20 \mathrm{~m}$ long and initially even small changes to the nozzle diameter could lead to instabilities. Despite difficulties, an all-important first hole was successfully drilled and a functioning first string deployed in that season. Table 1 shows the rapid increase in the number strings installed per season. Drilling became an engineering success of its own, with finely tuned operations performed by a well-trained and motivated drill crew.

Figure 3 shows the overlay of the depth-versus-time profiles of 20 holes drilled in one season. The average time for completing a hole of $\sim 55 \mathrm{~cm}$ diameter and 2,500 $\mathrm{m}$ depth was about 32 hours. In total, more than 900 tons of cargo and fuel have been delivered to the South Pole for IceCube. More than 300 Hercules LC-130 aircraft flights delivered the last leg of transportation from McMurdo to the South Pole over seven years. IceCube construction was organized in three shifts around the clock, with a total of 50 personnel on-ice from the middle of November to early February. It was clear that drilling would and should determine the schedule. That meant that more than 1,000 DOMs needed to be built, tested and shipped on time to meet the deadlines for vessel shipments. 

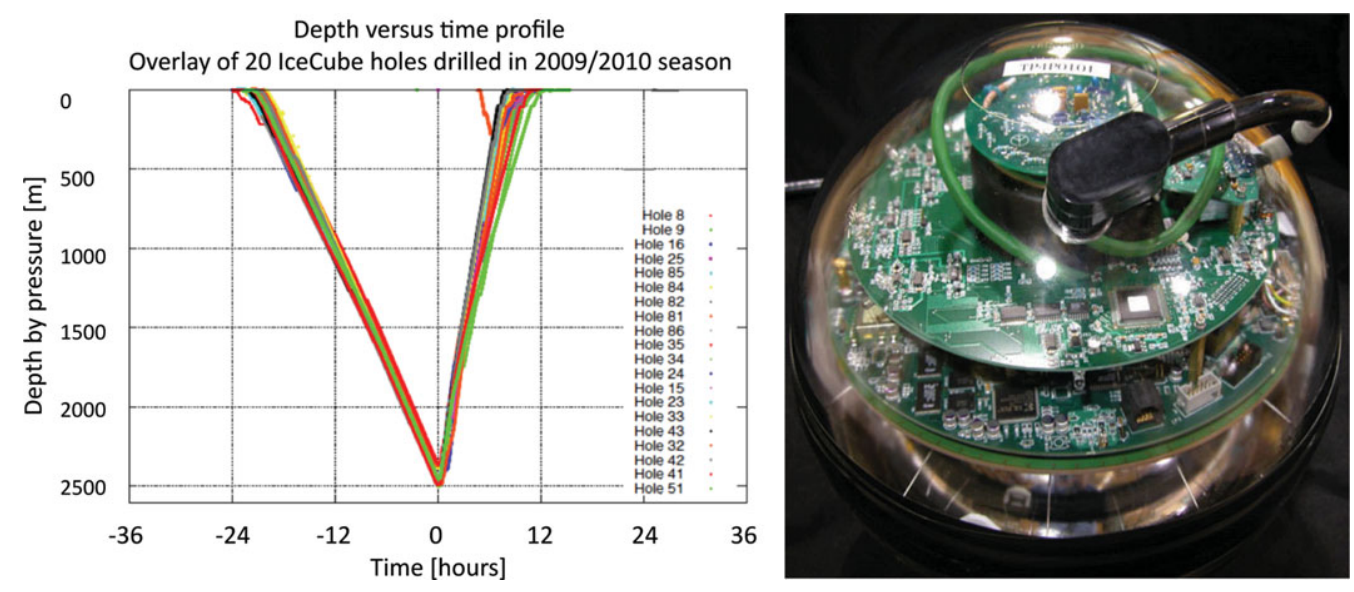

Figure 3. Left: The depth versus time profile is shown for 20 consecutive holes drilled with the IceCube Enhanced Hot Water Drill. The average drill time was 32 hours. Right: The IceCube Digital Optical Module; about $99 \%$ of the sensors are working since deployment in the ice.

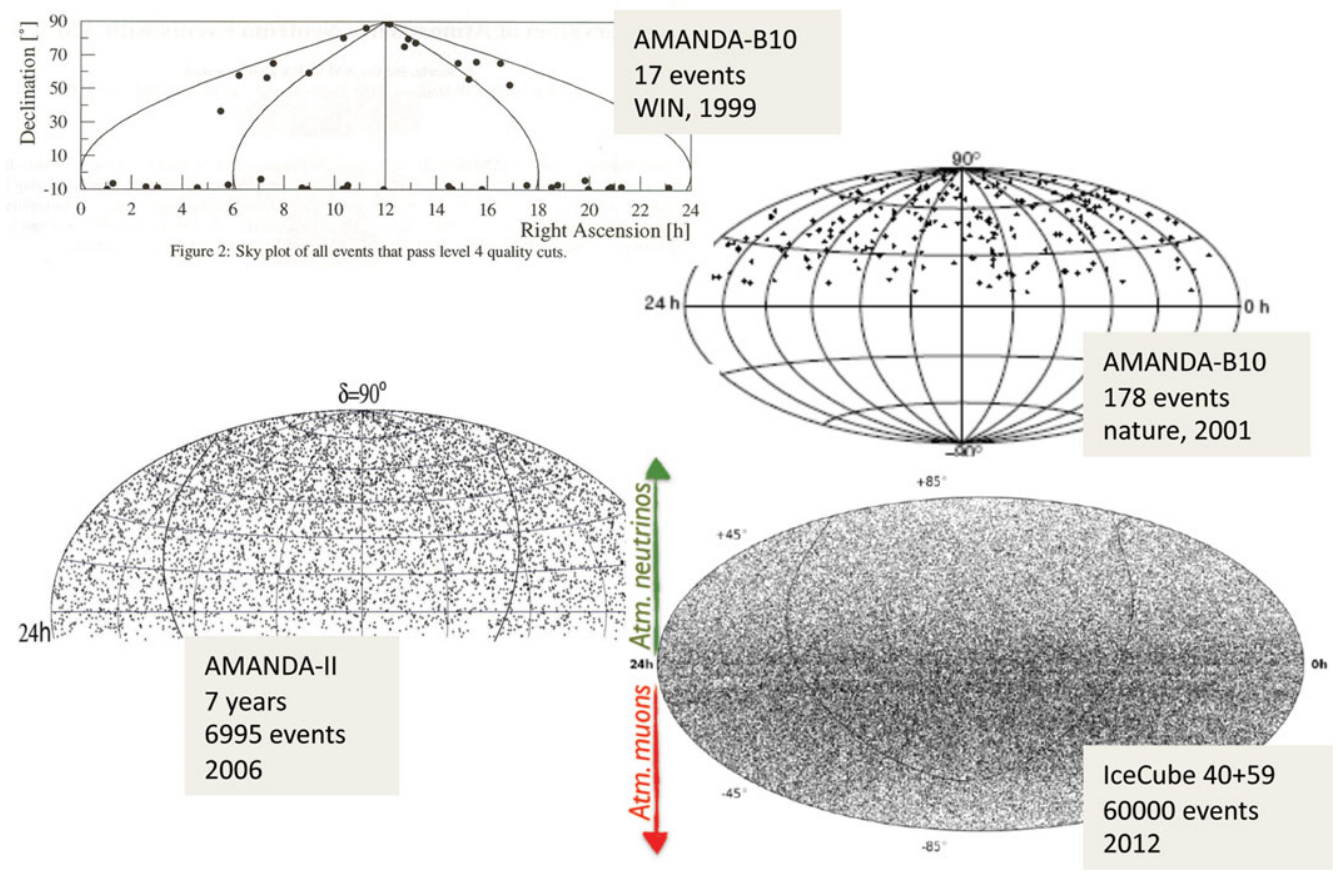

Figure 4. Skymaps at major steps of neutrinos astronomy as indicated in the figure.

The reliability and successful installation of the sensors was a critical requirement. In an initial count, only 84 sensors out of 5,160 did not commission successfully. The reliability after commissioning has been very high. Fewer than 20 sensors failed since commissioning during an accumulated lifetime of 20,000 sensor years. The rates appear to still be dropping. If one were to assume a constant failure rate, it would result in a mean time between sensor failures on the order of 1,000 years. 


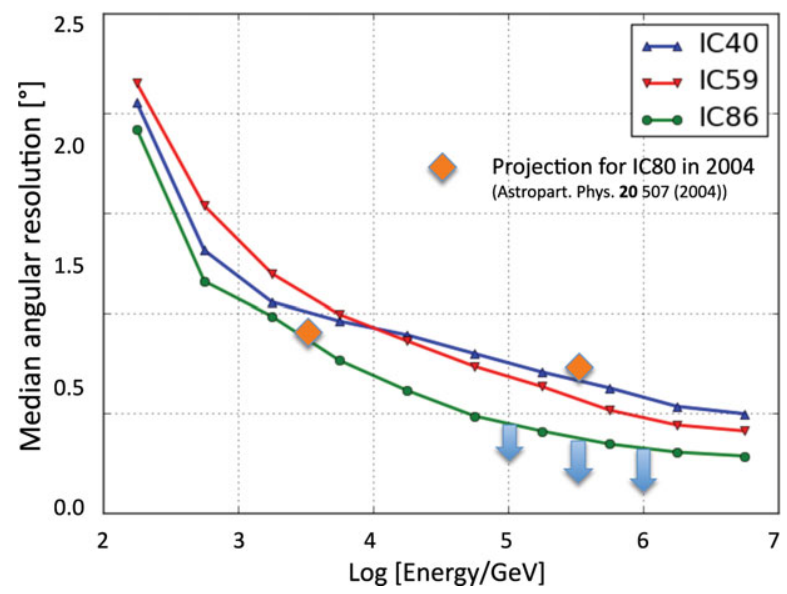

Figure 5. Angular resolution of IceCube as a function of energy. Further improvements are expected especially at higher energies.

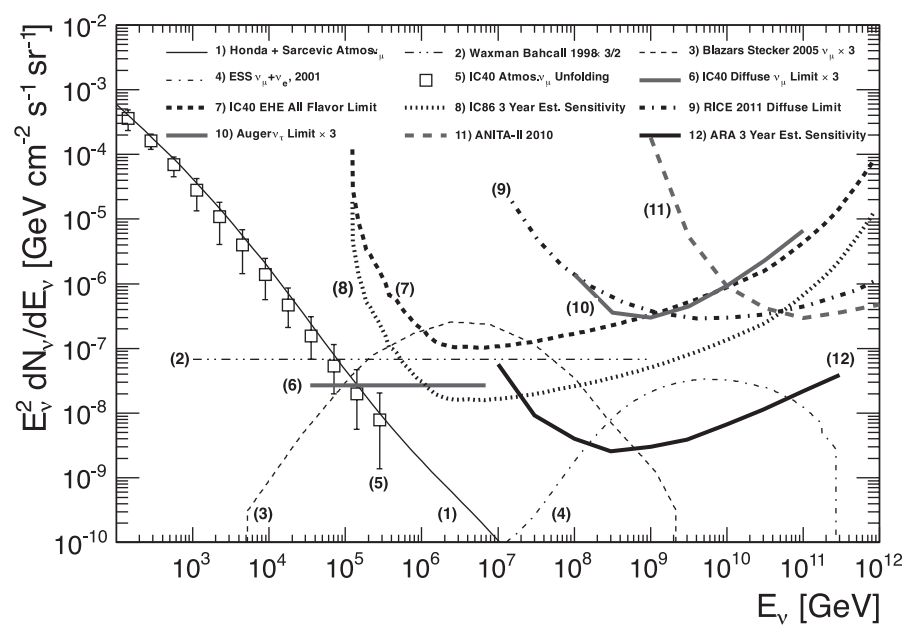

Figure 6. The measured atmospheric neutrino flux by IceCube is shown together with several predictions of neutrino fluxes and upper limits by experiments: (1) Atmospheric neutrino flux by Honda et al. (2007) + prompt by Sarcevic et al. (2008), (2) Diffuse neutrino flux (Waxman \& Bahcall 1995), (3) AGN Blazars (Stecker 2005), (4) Cosmogenic neutrino flux (Engel, Seckel \& Stanev 2001), (5) IceCube atmospheric neutrino flux unfolded measurement (Abbasi et al. 2011b), (6) IceCube 40, 1-year upper limit to diffuse neutrino flux (Abbasi et al. 2011a),

(7) IceCube 40, 1-year upper limit to extremely high-energy neutrinos (Abbasi et al. 2011c),

(8) IceCube 86, rough estimate of 3-year sensitivity (before this conference), (9) RICE upper limit (Kravchenko et al. 2012), (10) Auger 2-year limit x 3 (Auger 2012), (11) ANITA upper limit (Gorham et al. 2010), and (12) the Askaryan Radio Array (ARA) estimated 3-year sensitivity (Allison et al. 2012). Differential limits are corrected for energy binning and flavor differences.

Figure 4 shows the development of neutrino sky maps over 15 years of neutrino astronomy at the South Pole. The number of neutrinos per year increased from 17 to almost $10^{5}$ while the angular resolution improved from about $5^{\circ}$ to less then $0.4^{\circ}$. The angular resolution has been tested with real data using the Moon shadow of cosmic rays as a calibration source. Figure 5 shows the angular resolution for detector configurations of 40, 59 and 86 strings was a function of energy. Also shown are the originally projected resolution for IceCube, which has been well exceeded already. New reconstruction 
algorithms currently in development are expected to improve the resolution, especially at energies above $100 \mathrm{TeV}$, to levels $\sim 0.1^{\circ}$.

All performance parameters are meeting or exceeding initial expectations. The high rate of atmospheric muons of $3 \mathrm{kHz}$ is being processed at the IceCube laboratory in real time. A set of filters reduces the data rate from about 2,000 GB/day to a more manageable rate of $100 \mathrm{~GB} /$ day of events that are potentially interesting for physics searches. This data set is transmitted by satellite to the Northern Hemisphere on a daily basis. The sky maps offer only a superficial summary of the physics topics that can be covered with IceCube. The current state of measurements of atmospheric neutrinos by AMANDA and IceCube, some models for predictions of astrophysical neutrinos from GRBs and AGNs, as well as IceCube's limits on diffuse fluxes are shown in Figure 6. With 40 strings IceCube has measured the atmospheric neutrino spectrum up to energies of about $300 \mathrm{TeV}$, an energy where a hard component of astrophysical neutrinos is expected. At much higher energies, above $10^{17} \mathrm{eV}$, IceCube has already placed the best limits on the cosmogenic (GZK) neutrino flux.

\section{References}

Abbasi, R., et al. (IceCube Collaboration) 2012, Astropart. Phys. 35, 615

Abbasi, R., et al. (IceCube Collaboration) 2011a, ApJ 732, 18

Abbasi, R., et al. (IceCube Collaboration) 2011b, Phys. Rev. D 83, 012001

Abbasi, R., et al. (IceCube Collaboration) 2011c, Phys. Rev. D 83, 092003

Allison, P., et al. (ARA Collaboration) 2012, Astropart. Phys. 35, 457

Akhmedov, E. Kh., Razzaque, S., \& Smirnov, A. Yu. 2012, submitted; arXiv: 1205.7071

Andres, E., et al. (AMANDA Collaboration) 2001, Nature 410, 441

Askaryan, G. A. 1962, Soviet Physics, JTEP 14, 441

Askebjer, P., et al. (AMANDA Collaboration) 1995, Science 267, 1147

Auger Collaboration 2011, Phys. Rev. D 84, 122005; Erratum: Phys. Rev. D, 85, 029902(E)

Avrorin, A. V., et al. (Baikal Collaboration) 2009, Astron. Lett., 35, 651

Engel, R., Seckel, D., \& Stanev, T., 2001, Phys. Rev. D 64, 093010

Gaisser, T., these proceedings

Gorham, P. W., et al. (ANITA Collaboration) 2010, Phys. Rev. D 82, 022004

Honda, M., et al. 2007, Phys. Rev. D 75, 043006

Kravchenko, I., et al. 2012, Phys. Rev D, in press; arXiv:1106.1164

Lowder, D. M., et al. 1991, Nature 353, 331

Sarcevic, I., et al. 2008, Phys. Rev. D 78, 043005

Spiering, C. \& Katz, U., 2012, Prog. Part. Nucl. Phys. 67, 651

Stecker, F. W., 2005, Phys. Rev. D 72, 107301

Waxman, E. \& Bahcall, J., 1998, Phys. Rev. D 59, 023002

\section{Discussion}

QUESTION: What is the main difference between water and ice?

KARLE: The noise environment may be the biggest difference. The sensors in IceCube run at a noise rate of about $500 \mathrm{~Hz}$, and that is just the noise from the remnant radioactivity in the glass pressure housings of the sensors. This is why IceCube is sensitive to Supernova core collapse. The noise rate in the Mediterranean Sea is at the level of 100 to $1,000 \mathrm{KHz}$ for similar sensors, mostly from K40-decay and bioluminescence. Otherwise, absorption and scattering lengths are somewhat different, yet it does not make a big difference for detector designs and performance. 